\title{
Taste-potentiated color aversions in pigeons: Examination of CS preexposure and subsequent effects on postconditioning extinction of the taste aversion
}

\author{
TODD E. THIELE and JEROME FRIEMAN \\ Kansas State University, Manhattan, Kansas
}

\begin{abstract}
In the present study, we investigated the mechanisms of potentiation using pigeons as subjects. Half the groups were given unreinforced preexposures to the CS on 2 consecutive days before aversion training. After training, the taste aversion was extinguished in some of the groups conditioned with a colored, tasty compound. Postconditioning extinction of the taste aversion was found to attenuate potentiated color aversions. This was the case for the subjects that both were and were not preexposed to the CS. These results lend support to the summation theory of potentiation (Durlach $\&$ Rescorla, 1980) and weaken the claim that CS preexposures are necessary for obtaining such support. Alternative accounts of the data are discussed.
\end{abstract}

In the taste-aversion learning paradigm, when a less salient conditioned stimulus (CS) is paired with a more salient CS and this compound is followed by illness training, the typical finding is that the less salient CS evokes stronger aversive reactions (i.e., avoidance) than would have occurred if that CS alone had been followed by illness. This phenomenon is called potentiation, and it has been observed in a variety of situations.

In rats, it has been found that taste potentiates the aversive reaction to an odor (e.g., Droungas \& LoLordo, 1991; Durlach \& Rescorla, 1980; Kiefer, Rusiniak, \& Garcia, 1982; Lett, 1984; Miller, McCoy, Kelly, \& Bardo, 1986; Palmerino, Rusiniak, \& Garcia, 1980; Rusiniak, Hankins, Garcia, \& Brett, 1979; Westbrook, Homewood, Horn, \& Clarke, 1983), to visual properties of conditioned stimuli (Galef \& Osborne, 1978), to auditory properties of conditioned stimuli (Ellins, Cramer, \& Whitmore, 1985; Ellins \& von Kluge, 1987), to the conditioning environment (Best, Batson, Meachum, Brown, \& Ringer, 1985; Best \& Meachum, 1986; Miller et al., 1986 ), and to a second taste (Davis, Best, \& Grover, 1988; Davis et al., 1990; Kucharski \& Spear, 1985). In birds, it has been found that taste potentiates the aversive reaction to colored water (Franchina, Wright, Smith, Penn, \& Soeken, 1993; Jackson \& Fritsche, 1989; Lett, $1980,1984)$ and to colored food (Brett, Hankins, \& Garcia, 1976; Martin \& Lett, 1985).

Although potentiation has been demonstrated in a number of situations, the mechanisms that underlie po-

This experiment was presented at the 1993 annual meeting of the American Psychological Society in Chicago. Correspondence should be addressed to T. E. Thiele or J. Frieman, Department of Psychology, Bluemont Hall, Kansas State University, Manhattan, KS 66506-5302. tentiation are not well understood. Two theories have been proposed. Durlach and Rescorla (1980) suggested that potentiation is due to a summation of associations. When taste potentiates odor aversions, three associations are formed: a taste-toxin association, an odortoxin association, and a within-compound odor-taste association. During the odor-aversion test, the odor activates the representation of the toxin via the odor-toxin association and also activates the representation of the taste (via the odor-taste association), which in turn also activates the representation of the toxin. Thus, the representation of the toxin is activated twice, and this accounts for the potentiation phenomenon. Animals that receive odor alone associated with the toxin benefit only from the odor-toxin association. This theory predicts that if the taste-toxin association is extinguished after training, potentiation will be attenuated because the representation of the toxin will be activated only once, through the odor-toxin association.

A second theoretical account of potentiation, often referred to as associative potentiation (Lett, 1984), suggests that the presence of the taste during conditioning enhances the association between the weak CS (e.g., the odor) and the toxin (Garcia, Forthman Quick, \& White, 1984; Lett, 1984; Palmerino et al., 1980; Rusiniak et al., 1979). Garcia et al. (1984) described their version of this theory as a "sensory and-gate channeling system." According to this theory, taste belongs to the internal defense system and is readily associated with illness. Cues such as odors are part of the external defense system and are well associated with pain and fear, but these cues alone will not easily be associated with illness. When odor and taste are presented together before illness, the taste "gates" the odor into the internal defense system in such a way that it will be associated with the toxin; the 
organism will then demonstrate a conditioned aversion to odors. Unlike the summation theory, this theory suggests that it is not necessary to maintain the taste-toxin association after training in order to maintain the potentiated odor aversion.

There is evidence to support both theories. In the taste-potentiated odor-aversion paradigm, several authors have found that postconditioning extinction of the taste aversion attenuated associated odor aversions (Durlach \& Rescorla, 1980; Miller et al., 1986; Westbrook et al., 1983). Likewise, several authors have found that when one taste is used to potentiate the aversive reactions to a second taste, postconditioning extinction of one taste aversion attenuates the potentiated aversion to the second taste (Davis et al., 1988; Davis et al., 1990; Kucharski \& Spear, 1985). And in a paradigm in which a taste was used to potentiate environmental aversions, it has been found that postconditioning extinction of the taste aversion attenuated potentiated environmental aversions (Best et al., 1985). These data support the summation account of potentiation.

On the other hand, Lett (1984, Experiments 1 and 2) found that postconditioning extinction of the taste aversion did not attenuate potentiated odor aversions. Lett (1984, Experiment 3) and Franchina et al. (1993) also found that postconditioning extinction of the taste aversion did not attenuate potentiated color aversions. These data support the sensory and-gate channeling account of potentiation.

Lett (1984) suggested that conflicting evidence (specifically, between her data and those of Durlach \& Rescorla, 1980) was due to procedural differences-Durlach and Rescorla had presented their subjects with two unreinforced preexposures to the test solutions before training, whereas she did not use unreinforced preexposures. Lett suggested that these preexposures may be necessary to promote within-compound associations between the CS elements and would thus enhance the possibility of finding evidence in favor of the summation theory.

In two studies, researchers have directly tested the possibility that CS preexposure is responsible for the conflicting results. Davis et al. (1988) used one taste (denatonium saccharide) to potentiate the aversive reaction to another taste (saccharin) in rats. They found that postconditioning extinction of the denatonium saccharide aversion attenuated the potentiated saccharin aversion. This was demonstrated in two experiments; in one, the subjects were preexposed to the CS, and in the other they were not. Evidence favored the summation theory in both cases.

Droungas and LoLordo (1991) used taste to potentiate odor aversions in rats. In subjects that did not receive CS preexposures, postconditioning extinction of the taste aversion did not immediately attenuate the potentiated odor aversions; however, it was found that potentiated odor aversions were less resistant to extinction in a group whose taste aversion had been extinguished than they were in subjects that did not receive postconditioning extinction of the potentiating taste aversion (Experiment 2). On the other hand, when CS preexposures were administered, no potentiated odor aversions were learned, indicating latent inhibition. Droungas and LoLordo suggested that their data could not be explained by either the summation theory or the sensory and-gate channeling theory.

The present research, in which pigeons were used as subjects, was designed to further investigate Lett's suggestion that the use of CS preexposures by some experimenters may have produced inconsistent results in the past. To assess this possibility, half the subjects were given $\mathrm{CS}$ preexposures on 2 consecutive days before aversion training, and the other half were not. If the results show that postconditioning extinction of the taste aversion attenuated potentiated color aversions only in subjects that received CS preexposures, this would add evidence to Lett's suggestion that preexposures are necessary for the formation of within-compound associations and are thus necessary to obtain support for the summation theory.

\section{METHOD}

\section{Subjects}

The subjects were 51 homing pigeons of mixed sexes and various ages. They were individually housed and given ad-lib access to Purina Pigeon Chow and grit throughout the experiment (except during fluid presentation). The pigeons were exposed to a $16: 8-\mathrm{h}$ light:dark cycle.

\section{Materials}

McCormick green and red food colorings, both mixed at $1 \mathrm{ml}$ food coloring per liter of tap water, were used to provide the colored waters. Vinegar water was made by adding $10 \mathrm{ml} \mathrm{Heinz} \mathrm{dis-}$ tilled vinegar to 1 liter of tap water. When red vinegar water was mixed, $1 \mathrm{ml}$ of McCormick red food coloring and $10 \mathrm{ml}$ of Heinz distilled vinegar per liter of tap water were used. Salt water was made by mixing $7.0 \mathrm{~g}$ of $\mathrm{NaCl}$ to 1 liter of tap water. Green salt water was made by combining $1 \mathrm{ml}$ of McCormick green food coloring, $7.0 \mathrm{~g} \mathrm{NaCl}$, and 1 liter of tap water. The solutions were administered to the birds in 9-oz. clear plastic tumblers that were suspended on the cage fronts. The unconditioned stimulus was $0.3 \mathrm{M}$ lithium chloride, administered at $2 \mathrm{ml}$ per $100 \mathrm{~g}$ of body weight; these were the same concentrations used by Lett (1984).

\section{Procedure}

Throughout the experiment, the food containers were always removed $30 \mathrm{~min}$ before fluid access; fluid was administered at the same time each day and was presented for $15 \mathrm{~min} /$ day (except where deviations from this procedure are noted). The pigeons were first acclimated to a $15-\mathrm{min} /$ day drinking schedule over a 4-day period. The birds were then divided into six groups on the basis of their average water consumption (in grams) from the last 3 days of acclimation. Groups PC $(n=9)$, PCT $(n=9)$, and PCT-E $(n=8)$ received 2 days of unreinforced $\mathrm{CS}$ preexposure, and Groups $\mathrm{C}$ $(n=8)$, CT $(n=9)$, and CT-E $(n=8)$ did not receive CS preexposure. The experimental procedure was then subdivided into five phases: pretraining, preexposure, training, extinction, and testing. The solutions that the groups received at each phase are presented in Table 1 . 
Table 1

Solutions Presented to Each Group by Phase of the Experiment

\begin{tabular}{lclllll}
\hline & \multicolumn{5}{c}{ Phase } \\
\cline { 2 - 6 } \multicolumn{1}{c}{ Group } & Pretraining & Preexposure & Training & Extinction & Testing & Posttest \\
\hline Preexposure & Vin & Red & Red & Vin & Red & \\
PC & Grn & Red Vin & Red Vin & Salt & Red & Vin \\
PCT & Grn & Red Vin & Red Vin & Vin & Red & Vin \\
PCT-E & & & & & & \\
No Preexposure & Vin & Vin & Red & Vin & Red & \\
C & Grn & Grn Salt & Red Vin & Salt & Red & Vin \\
CT & Grn & Grn Salt & Red Vin & Vin & Red & Vin \\
CT-E & & & & &
\end{tabular}

Note-Grn, green; Vin, vinegar.

Pretraining. To acclimate the birds to drinking novel solutions, they were given access to such solutions for 1 day prior to the test solution preexposure phase. All the groups were given solutions that were not involved in the training phase. Groups $\mathrm{PC}$ and $\mathrm{C}$ received access to vinegar water on this day, and Groups PCT, PCT-E, CT, and CT-E received access to green-colored water.

Preexposure. During the following 2 days, half the groups were preexposed to the solutions they would receive during the training phase. Group PC received access to red-colored water, and Groups PCT and PCT-E received access to red vinegar water. The following groups were not preexposed to their training solutions and were given additional access to novel stimuli to further eliminate neophobia: Group $\mathrm{C}$ received access to vinegar water, and Groups CT and CT-E received access to green salt water.

Training. On the training day, the food containers were removed from the birds' cages $2 \mathrm{~h}$ before conditioning; this was done to avoid any food-toxin associations. On this day, Groups PC and $\mathrm{C}$ received access to red-colored water, and Groups PCT, PCT-E, CT, and CT-E received access to red vinegar water. Immediately after the 15 -min access to the solutions, the birds were given intraperitoneal injections of lithium chloride. Four hours after training, food and water were made available to the birds for 5 days, during which time they were allowed to recover. The symptoms that accompanied illness training were vomiting in most of the birds and shivering and diarrhea in all the birds.

Extinction. Following the 5-day recovery period, all the birds were again acclimated to the $15-\mathrm{min} /$ day drinking schedule over a 2-day period. Over the next 3 days, Groups PCT-E and CT-E received unreinforced exposure to vinegar water to extinguish the taste aversion. To assess the progress of extinction, Groups PC and $\mathrm{C}$ (neither of which consumed the vinegar water on the training day) were also given access to vinegar water. The extinction phase was carried out until there were no statistical differences between the groups that were going through taste-aversion extinction (PCT-E and CT-E) and the comparison groups (PC and C). On Day 1 of the extinction phase, these groups were given 15-min access to vinegar water. One hour later, these groups were given 24-h access to the solution. On Day 3 of the extinction phase, these groups were again given 15 -min access to the vinegar water. During this phase, Groups PCT and CT received access to salt water, and they were administered the solutions at the same times as those for the above groups.

Testing. On the day after the extinction phase, all the groups received access to red-colored water in order to test the degree of colored-water aversions. On the next day, Groups PCT, PCT-E, CT, and CT-E received access to vinegar water. This posttest was done in an attempt to demonstrate attenuation of the taste aversions in the potentiation-trained groups that went through the tasteextinction procedure relative to that of the potentiation-trained groups that did not receive that treatment.

\section{RESULTS}

All statistical analyses (including Newman-Keuls tests) were evaluated at $p=.05$. The dependent variable tested in all cases was the amount of fluid consumed measured in grams.

\section{Pretraining, Preexposure, Training, and Extinction}

A one-way analysis of variance (ANOVA) comparing all six groups was run on the pretraining data; no significant differences were found. A mixed-factor (groups $X$ days) ANOVA was run on the preexposure data; neither the main effects nor the interaction effect was significant. A one-way ANOVA was run to compare all groups on the training day; again, no significant differences were found.

The results from the extinction phase are presented in Figure 1. A mixed-factor (preexposure $\times$ groups $\times$

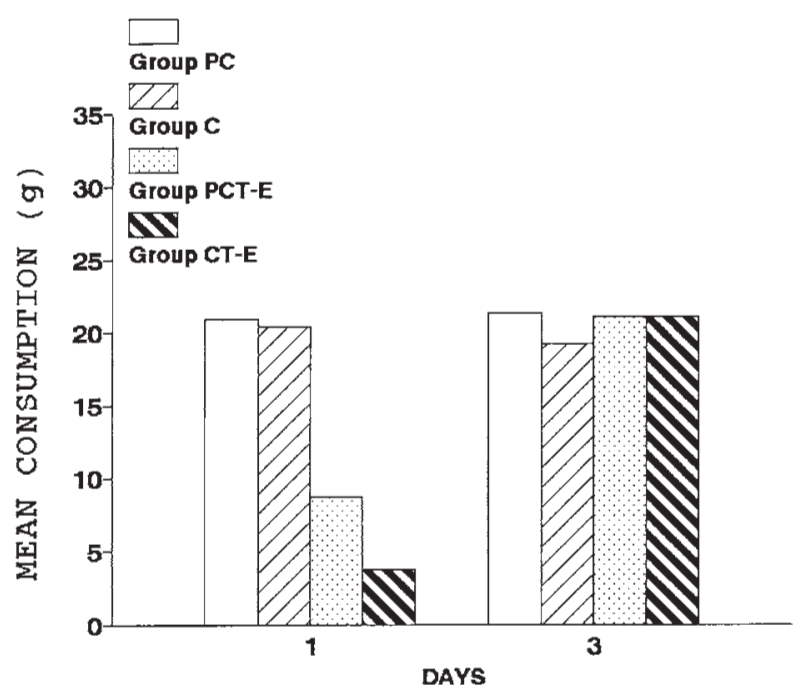

Figure 1. Mean fluid consumption (in grams) from the 15-min access to vinegar water in the extinction phase. Data from the first 15-min access from Day 1 of the extinction phase are on the left side of the figure, and those from the second 15-min access from Day 3 are on the right. Relative to the comparison groups ( $\mathrm{PC}$ and $\mathrm{C}$ ), taste aversions were extinguished in Groups PCT-E and CT-E by the second 15-min access period. 
days) ANOVA was performed to examine consumption of vinegar water during the 15 -min fluid-access period from Day 1 of the extinction phase and the second 15-min fluid-access period that was given on Day 3 of the extinction phase (data from the 24-h fluid-access period were not included in the analysis). The first betweensubjects factor (preexposure) involved a comparison of the groups that received CS preexposure ( $\mathrm{PC}$ and PCT-E) with those that did not (C and CT-E), and the second between-subjects factor (groups) involved a comparison of the groups that went through extinction (PCT-E and CT-E) with the comparison groups (PC and C). A within-subjects factor (days) involved a comparison of consumption during the 15-min fluid-access period on Day 1 of the extinction phase with consumption during the 15-min fluid-access period on Day 3 of the extinction phase. Neither the preexposure main effect nor the groups main effect reached statistical significance. However, the main effect of days was significant: overall, consumption increased from Day 1 to Day 3 of the extinction phase $[F(1,29)=13.82]$. The interaction between the groups effect and the days effect was also significant $[F(1,29)=15.52]$. Post hoc (NewmanKeuls) tests revealed that at the first 15 -min access period, the comparison groups consumed significantly more vinegar water than the groups going through extinction. However, at the second 15-min access period, there was no significant difference in consumption between these groups. No other interactions were significant.

\section{Testing}

The mean fluid consumption for all the groups over the colored-water test phase is presented in Figure 2. A

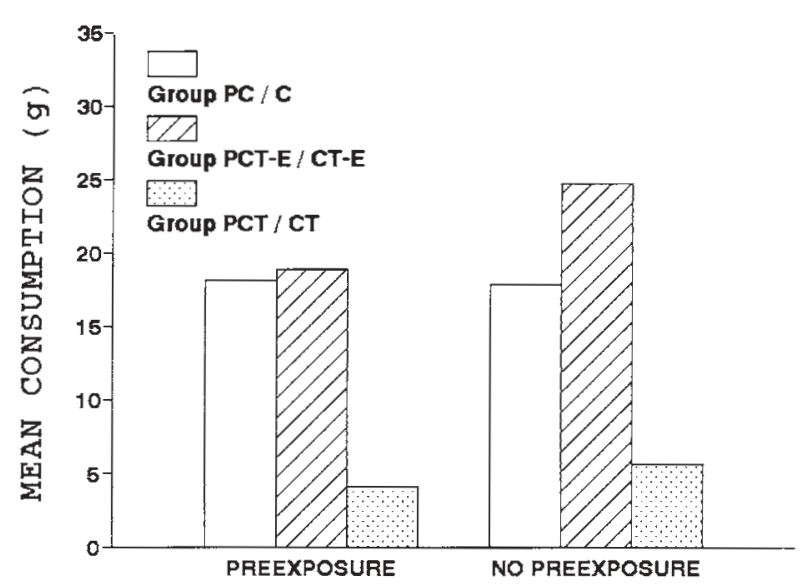

Figure 2. Mean fluid consumption (in grams) from the coloredwater test phase. Data from groups that received CS preexposure are on the left side of the figure (PC, PCT-E, and PCT), and data from groups that did not receive CS preexposure are on the right (C, CT-E, and $C T$ ). Groups $P C$ and $C$ were trained with red-colored water, and Groups PCT-E, PCT, CT-E, and CT were trained with red vinegar water. Groups PCT-E and CT-E received postconditioning extinction of the taste aversion. two-way (preexposure $\times$ groups) ANOVA was run on the data. The first factor (preexposure) involved a comparison of the groups that received CS preexposures with those that did not, and the second factor (groups) involved a comparison of the colored-water-trained groups ( $\mathrm{PC}$ and $\mathrm{C})$, the potentiation-trained groups (PCT and CT), and the potentiation-trained groups that received taste-aversion extinction (PCT-E and CT-E). The main effect of preexposure was not significant. There was a significant difference in fluid consumption between the various treatment groups $[F(2,45)=9.53]$. A Newman-Keuls test revealed that the subjects that received potentiation training and no postconditioning extinction of the taste aversion (data collapsed across Groups PCT and CT) drank significantly less redcolored water than all the other subjects. There were no significant differences between the subjects that received red-colored water during training (data collapsed across Groups PC and C) and those that received potentiation training and postconditioning extinction of the taste aversion (data collapsed across Groups PCT-E and CT-E). The interaction between the two factors was not significant.

A two-way (preexposure $\times$ groups) ANOVA was run on the data from the final vinegar-water test. The first factor (preexposure) involved a comparison of the groups that received CS preexposures with those that did not, and the second factor (groups) involved a comparison of the groups that received postconditioning extinction of the taste aversion (Groups PCT-E and CT-E) with those that did not (Groups PCT and CT). The main effect of preexposure was not significant. The groups that received postconditioning extinction of the taste aversion drank more vinegar water $(M=24.65 \mathrm{~g}$, when collapsed across Groups PCT-E and CT-E) than the groups that $\operatorname{did} \operatorname{not}(M=7.41 \mathrm{~g}$, when collapsed across Groups PCT and CT); the difference was significant $[F(1,30)=$ 28.93]. There was no significant interaction between the two factors.

\section{DISCUSSION}

The data of principal interest are those from the colored-water test phase. The only groups that demonstrated a potentiated color aversion relative to the colored-water-trained groups ( $\mathrm{PC}$ and $\mathrm{C}$ ) were those that had received potentiation training and no postconditioning extinction of the taste aversion (PCT and CT). There were no significant differences between the colored-water-trained groups and the groups that had received potentiation training followed by extinction of the taste aversion (PCT-E and CT-E). This suggests that extinction of the taste aversions attenuated the potentiated color aversions. Because extinction of the taste aversion also attenuated the color aversion, the presence of within-compound associations between the color and taste elements may be inferred.

One objective of the present study was to test Lett's (1984) suggestion that CS preexposure may be neces- 
sary for the formation of within-compound associations (i.e., color-taste associations). This question was examined by comparing the subjects that had received CS preexposure with those that had not. It was found that postconditioning extinction of the taste aversions attenuated potentiated color aversions - with or without unreinforced preexposures to the CS. These data lend support for the summation theory, which states that the taste aversion is necessary for potentiation. Thus, the use of CS preexposure by some experimenters may not be the factor responsible for previous inconsistent results when taste aversions are extinguished after potentiation training.

The main purpose of examining the data from the pretraining, preexposure, and training phases was to demonstrate the compatibility of fluid consumption between the groups. There were no significant differences between the groups at these phases, indicating that all the groups shared a similar base fluid-consumption level. At the first 15-min fluid-access period during the extinction phase, the groups that had received red-vinegar-toxin pairings (PCT-E and CT-E) demonstrated significantly stronger avoidance of vinegar water than did the comparison groups ( $\mathrm{PC}$ and $\mathrm{C}$ ) that had never received vinegar-toxin pairings. However, there were no differences between these groups at the second 15-min fluidaccess period, suggesting that taste aversions had been extinguished in Groups PCT-E and CT-E. The final vinegar-water test indicated that the groups that went through vinegar-water extinction consumed significantly more vinegar water than the groups that did not receive this treatment (PCT-E and CT-E vs. PCT and $\mathrm{CT})$. Together, the results from the extinction and vinegar-water-consumption phases indicate that the taste aversions were significantly attenuated in Groups PCT-E and CT-E.

In the present study, we employed procedures very similar to those used by Lett (1984, Experiment 3), but we added CS preexposures in some cases. It is therefore difficult to account for the inconsistent results between the present study and Lett's earlier study. Contrary to the present study, Lett found that extinction of the tastetoxin association did not attenuate potentiated color aversions. It could be argued that, in the present study, the effects of the green salt solution given to the nonpreexposed potentiation-trained groups (CT and CT-E) during the preexposure phase generalized to the red vinegar solution used during conditioning. This may seem likely, given that the preexposed and nonpreexposed groups drank similar amounts of fluid during the conditioning phase. If this were the case, all the groups would have been preexposed to the conditioned stimulus before training, thus making procedures from the present experiment different from those used by Lett in all cases. This is a possibility; however, it could also be argued that 2 days of exposure to the green salt solution would likely make it a familiar stimulus and thus would allow the subjects to discriminate it from the red vinegar solution. Furthermore, the similarity in con- sumption of the red vinegar solution between the preexposed and nonpreexposed groups during the training phase may indicate attenuated neophobia to novel stimuli in Groups CT and CT-E due to exposure to the green salt solution.

Although postconditioning extinction of the taste aversion attenuated potentiated color aversions in the present study, caution is necessary when concluding that these results support the summation theory. Westbrook et al. (1983) found that postconditioning extinction of a taste aversion attenuated potentiated odor aversions in rats; however, they also found that extinction of a taste aversion not associated with the odor also attenuated the potentiated odor aversion (Experiment 4). They suggested that postconditioning extinction of a taste aversion-either the taste conditioned with the odor or the taste conditioned alone-may degrade the representation of the US (toxin). With such an interpretation, it is possible that attenuation of potentiated odor aversions after taste-aversion extinction may not be modulated by within-compound associations; rather, they may be directly modulated via an odor-US representation association. However, the summation theory can also account for Westbrook et al.'s (1983) data as they interpret it: When presented to the subject after taste-aversion extinction, the odor would activate the representation of the US twice, but potentiated odor aversions would not be observed because the US representation would be degraded.

LoLordo and Droungas (1989) offered an alternative interpretation of Westbrook et al.'s (1983) results. They noted the possibility of generalization between the taste paired with odor before conditioning and the taste conditioned alone. If this is the case, extinguishing aversions to the independently conditioned taste should also extinguish aversions to the taste conditioned with the odor. Attenuated odor aversions could thus be attributed to within-compound associations.

It is possible that there was generalization between the color and taste elements used in the present experiment. Thus, when extinguishing the taste-toxin association, the color-toxin association may have been extinguished as well, and this attenuated the potentiated color aversions. Accounting for attenuated color aversions in these terms does not rely on within-compound associations. However, given that the aversion to vinegar in the groups conditioned after consumption of this element was significantly stronger than that in the groups trained with color alone (see Figure 1), it seems safe to conclude that any generalization between the colored stimulus and vinegar is minimal.

Finally, the factor(s) responsible for past inconsistent results are at present unknown. However, there seems to be mounting evidence in support of the summation theory (Durlach \& Rescorla, 1980) over the sensory andgate channeling account of potentiation (Garcia et al., 1984) when taste aversions are extinguished after potentiation training. 


\section{REFERENCES}

Best, M. R., Batson, J. D., Meachum, C. L., Brown, E. R., \& RINGER, M. (1985). Characteristics of taste-mediated environmental potentiation in rats. Learning \& Motivation, 16, 190-209.

Best, M. R., \& Meachum, C. L. (1986). The effects of stimulus preexposure on taste-mediated environmental conditioning: Potentiation and overshadowing. Animal Learning \& Behavior, 14, 1-5.

Brett, L. P., Hankins, W. G., \& Garcia, J. (1976). Prey-lithium aversions: III. Buteo hawks. Behavioral Biology, 17, 87-98.

Davis, S. F., Best, M. R., \& Grover, C. A. (1988). Toxicosis-mediated potentiation in a taste/taste compound: Evidence for withincompound associations. Learning \& Motivation, 19, 183-205.

Davis, S. F., Best, M. R., Grover, C. A., Bailey, S. A., Freeman, B. L., \& MAYLEBEN, M. A. (1990). The effects of taste extinction on ingestional potentiation in weanling rats. Animal Learning \& Behavior, 18, 444-452.

Droungas, A., \& LoLordo, V. M. (1991). Taste-mediated potentiation of odor aversion induced by lithium chloride: Effects of preconditioning exposure to the conditioned stimulus and postconditioning extinction of the taste aversion. Learning \& Motivation, 22 , 291-310.

Durlach, P. J., \& Rescorla, R. A. (1980). Potentiation rather than overshadowing in flavor-aversion learning: An analysis in terms of within-compound associations. Journal of Experimental Psychology: Animal Behavior Processes, 6, 175-187.

Ellins, S. R., Cramer, R. E., \& Whitmore, C. (1985). Taste potentiation of auditory aversions in rats (Rattus norvegicus): A case for spatial contiguity. Journal of Comparative Psychology, 99, 108-111.

Ellins, S. R., \& von Kluge, S. (1987). Preexposure and extinction effects of lithium chloride induced taste-potentiated aversions for spatially contiguous auditory food cues in rats. Behavioral Neuroscience, 101, 164-169.

Franchina, J. J., Wright, D. J., Smith, R. F., Penn, C. Y., \& Soeken, E. (1993). Extinction of taste aversion does not eliminate taste-mediated aversion to visual cues: Replicating Lett (1984). Bulletin of the Psychonomic Society, 31, 426-428.

Galef, B. G., \& Osborne, B. (1978). Novel taste facilitation of the association of visual cues with toxicosis in rats. Journal of Comparative \& Physiological Psychology, 92, 907-916.

Garcia, J., Forthman Quick, D., \& White, B. (1984). Conditioned disgust and fear from mollusk to monkey. In D. L. Alkon \& J. Farley (Eds.), Primary neural substrates of learning and behavior change (pp. 47-61). Cambridge: Cambridge University Press.

JACKSON, R. L., \& Fritsche, M. B. (1989). Potentiation and overshadowing in pigeons. Learning \& Motivation, 20, 15-35.

Kiefer, S. W., RusiniaK, K. W., \& Garcia, J. (1982). Flavor-illness aversions: Gustatory neocortex ablations disrupt taste but not tastepotentiated odor cues. Journal of Comparative \& Physiological Psychology, 96, 540-548.

Kucharski, D., \& Spear, N. E. (1985). Potentiation and overshadowing in preweanling and adult rats. Journal of Experimental Psychology: Animal Behavior Processes, 11, 15-34.

LETT, B. T. (1980). Taste potentiates color-sickness associations in pigeons and quail. Animal Learning \& Behavior, 8, 193-198.

LETT, B. T. (1984). Extinction of taste aversion does not eliminate taste potentiation of odor aversion in rats or color aversion in pigeons. Animal Learning \& Behavior, 12, 414-420.

LoLordo, V. M., \& Droungas, A. (1989). Selective associations and adaptive specializations: Taste aversions and phobias. In S. B. Klein \& R. R. Mowrer (Eds.), Contemporary learning theories: Instrumental conditioning theory and the impact of biological constraints on learning (pp. 145-179). Hillsdale, NJ: Erlbaum.

Martin, G. M., \& LetT, B. T. (1985). Formation of associations of colored and flavored food with induced sickness in five avian species. Behavioral \& Neural Biology, 43, 223-237.

Miller, J. S., McCoy, D. F., Kelly, K. S., \& Bardo, M. T. (1986). A within-event analysis of taste-potentiated odor and contextual aversions. Animal Learning \& Behavior, 14, 15-21.

Palmerino, C. C., Rusiniak, K. W., \& Garcia, J. (1980). Flavorillness aversions: The peculiar roles of odor and taste in memory for poison. Science, 208, 753-755.

Rusiniak, K. W., Hankins, W. G., Garcia, J., \& Brett, L. P. (1979). Flavor-illness aversions: Potentiation of odor by taste in rats. $\mathrm{Be}$ havioral \& Neural Biology, 25, 1-17.

Westbrook, R. F., Homewood, J., Horn, K., \& Clarke, J. C. (1983). Flavour-odour compound conditioning: Odour-potentiation and flavour-attenuation. Quarterly Journal of Experimental Psychology, 35B, 13-33.

(Manuscript received July 13, 1993; revision accepted for publication March 9, 1994.) 